increased intake of ascorbic acid during the polar winter. (It may be recalled that the death of Captain Scott and his companions has recently been ascribed once more to scurvy.)

Medically, the Antarctic seems to provide an environment that is dangerous because of extreme cold, winter darkness and isolation, but safe from the point of view of microorganisms which would seem to be almost absent from this sterile continent. D. J. Lugg (Australia), P. Doury and S. Pattin (France) and R. M. Lloyd (Britain) described methods of medical selection, the causes of illness, and so on. Accidents are, in all cases, the most important category, both in number and severity. In spite of the great changes in the technology of Antarctic life and travel, it is far from being a safe place, and the mortality rate in a young and highly selected group is substantial.

The sterility of Antarctica presents the microbiologists both with opportunities and challenges. When a ship leaves a group of men at an Antarctic base they are, in most cases, marooned for nearly a year. For one or two weeks they will have coughs and colds, then they are usually quite free of infection until a year or so later when they are relieved; most of them then develop severe colds. Does the antibody level decline during the period of isolation? T. R. Allen and M. J. Holmes (Britain) have shown that this does not occur, and the severity and frequency are presumably due to exposure to one of the very large number of viruses responsible, to which the individuals concerned have not become immune.

Low environmental temperature is the most obvious characteristic of the Antarctic environment, but there is also the long polar night and the long polar day, and the isolation of small groups at the various stations maintained by as many as twelve nations. The effects of these factors on human behaviour were described in a part of the symposium organized by E. K. E. Gunderson (USA), who outlined the selection techniques he and his colleagues have developed. The chief factors concerned are emotional stability, task compatibility and social compatibility. At first, selection tests correlated poorly with performance in the field, but effective selection tests have gradually been evolved and are now proving to be useful. L. Crocq (France) considers there had been an increase in psychological problems at French bases as they had become more comfortable, but I. Tikhomirov (USSR) thinks that both medical and psychological difficulties diminished at the Russian stations with successive physical improvements. J. Shurley (USA) emphasized the value of polar stations for behavioural studies, as observations in a confined situation over a period of a year could and did yield a mass of information that is difficult to obtain in any other way. He also described the results of studies on sleep at the south polar station.

Sleep disturbance is frequently reported by United States personnel, and is described as "big-eye". It has been found that the extent of stage 4 sleep, the "deepest" level, gradually diminished and, after approximately six months at the south pole, was almost absent. Even more remarkably, on return to the United States stage 4 sleep remained at a very low level for periods up to a year. At present, it is not clear whether this changed pattern is due to a combination of the effects of the polar environment and the effects of the high altitude of the south pole. Some of the physiological changes observed both at the south polar station and at Vostock, the USSR station on the plateau, are clearly due to altitude, but more work is evidently needed to identify the combined effects of altitude, extreme cold, isolation and the changing pattern of light and darkness.

There were a number of papers dealing with studies in Arctic regions, specifically Alaska, Canada and Norway, and, at an informal discussion, ways in which Arctic-Antarctic collaboration could be developed were debated.

\section{MOLECULAR BIOLOGY

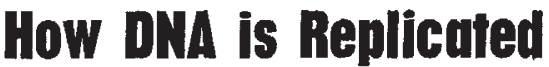

from our Molecular Genetics Correspondent LAST week, I discussed progress in showing that episome replication in Escherichia coli is under the same sort of control as that of the bacterial chromosome. Several other articles in the September issue of the Proceedings of the National Academy of Sciences also illustrate the concern of molecular biologists to solve the central problem of how DNA is replicated. On page 2570, Olsen, Staudenbauer and Hofschneider show that $d n a B$ mutants of $E$. coli cannot replicate the double stranded form of phage M13 DNA, although they can convert the infective single strands to replicative double strands. DnaB mutants appear to be defective in some cellular property concerned with the cell membrane, which lends further support to the idea that replication of M13 DNA involves membrane bound intermediates.

Also concerned with the $\operatorname{dnaB}$ mutants, Schekman et al. (ibid., 2691) present the latest of their series of articles on the role of RNA in initiating replication of DNA. Their previous work has concentrated on phage M13, replication of which is inhibited by the addition of rifampicin, an inhibitor of RNA synthesis. They now turn their attention to $\phi \mathrm{X} 174 \mathrm{DNA}$, replication of which is not inhibited by rifampicin, in vitro or in vivo.

The single stranded form of the phage can be converted to double strands in a cell-free extract system, but extracts of the dnaA and $d n a B$ mutants that are sensitive to temperature are inactive at high temperature. A combination of the extracts is active, which suggests that the $d n a A$ and $d n a B$ mutations affect different enzymes. This system is proving useful as a means to isolate the $d n a A$ and $d n a B$, by purifying the extracts of one mutant strain which

\title{
ATP Hydrolysis and Restriction Endonuclease
}

IN Nature New Biology next week (November 8) Yuan, Heywood and Meselson report experiments which lead to the interesting speculation that the hydrolysis of ATP to ADP is required to maintain Escherichia coli. $\mathrm{K}$ restriction endonuclease in a configuration which enables this enzyme to recognize its substrate, unmodified DNA. E. coli $\mathbf{K}$ restriction endonuclease cleaves DNA which has not been modified by replication in $E$. coli $\mathrm{K}$ cells. For example it cleaves unmodified lambda phage DNA into five roughly equally sized pieces and of course this enzyme provides $E$. coli $\mathbf{K}$ cells with a defence against foreign DNAs.

Yuan et al., who found that the activity of this endonuclease depends amongst other things on a supply of ATP, noticed that as the endonuclease is purified an ATPase activity purifies with it. This ATPase has the same $p H$ optimum as the endonuclease activity and similar requirements, including the presence of unmodified DNA. In other words one molecule seems to have two enzymatic activities, an ATPase activity as well as an endonuclease activity. Moreover, they noticed that the hydrolysis of ATP continues long after nucleolytic attack of DNA has ceased, and about $10^{5}$ ATP molecules are hydrolysed for every lambda DNA molecule that is cleaved.

Why does this endonuclease require to hydrolyse ATP if it is to cleave unmodified DNA, and why are so many ATP molecules hydrolysed? The energy released by the hydrolysis of so many ATP molecules is unlikely to be required for the nucleolytic reaction, not least because many other nucleases do not show this requirement. Yuan et al. suggest that the energy provided by the ATP may be required to maintain the endonuclease in an unstable configuration in which it can recognize its substrate. 